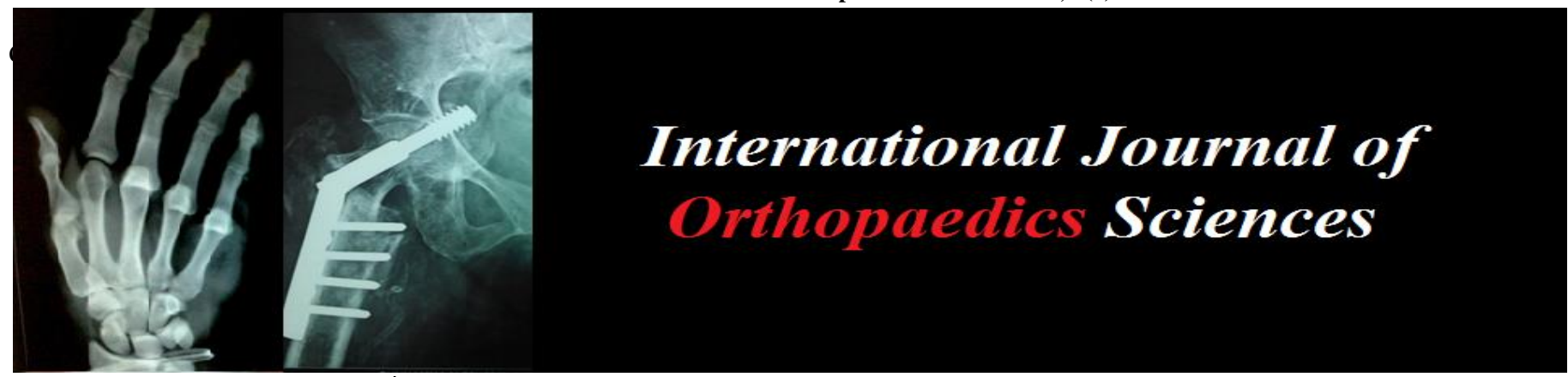

E-ISSN: 2395-1958

P-ISSN: 2706-6630

IJOS 2021; 7(3): 689-691

(C) 2021 IJOS

www.orthopaper.com

Received: 25-05-2021

Accepted: 27-06-2021

Dr. Sai Kumar Reddy

Mukkamalla

Registrar, Ortho One Orthopedic Speciality Center, Coimbatore,

Tamil Nadu, India

Dr. P Yuvarajan

Joint Replacement Surgeon,

Ortho One Orthopedic Speciality

Center, Coimbatore, Tamil Nadu,

India

Dr. B Vidya Sagar

Foot and Ankle Surgeon,

Ortho One Orthopedic Speciality

Center, Coimbatore, Tamil Nadu, India
Corresponding Author Dr. Sai Kumar Reddy Mukkamalla

Registrar, Ortho One Orthopedic Speciality Center, Coimbatore,

Tamil Nadu, India

\section{Extraarticular deformity of the right knee with $b / l$ varus corrected in a single stage: A case report and review of literature}

\author{
Dr. Sai Kumar Reddy Mukkamalla, Dr. P Yuvarajan and Dr. B Vidya \\ Sagar
}

DOI: https://doi.org/10.22271/ortho.2021.v7.i3j.2821

\section{Abstract}

Total Knee Arthroplasty has enjoyed substantial favourable outcomes of late with the advent of new techniques and implantation. The procedure of choice - Total Knee Replacement becomes challenging when a patient is suffering from systemic diseases and severe anatomical deformities. Presented here is the case report of a 64-year-old female with known Rheumatoid Arthritis and severe extra articular deformity adding to her severe varus deformity thereby compromising her quality of life. We corrected the deformity followed by total knee arthroplasty in a single setting, imbued with the hope that the procedure had on offer for the said patient, a tremendous improvement in quality of life.

Keywords: total knee arthroplasty, extra articular deformity, rheumatoid arthritis, varus deformity and single stage

\section{Introduction}

The course of rheumatoid arthritis varies from mild disease to severe joint destructive variant that progresses rapidly, eventually leading to unremitting pain and joint deformity in early ages. In advanced disease, total knee arthroplasty has proven to be the most successful intervention that reduces knee pain, assures deformity correction and improves functional outcomes in rheumatoid arthritis patients ${ }^{[1]}$.

Case Report: A 64-year-old female patient with BMI of 22.2, beset by rheumatoid arthritis and hypertension came to our Centre with severe b/l knee pain unable to walk or stand in the last three years. On examination, we diagnosed her with severe varus deformity on both sides. Preliminary investigations also revealed a malunited proximal tibia fracture on the right side and severe pain on the left side. At first, we addressed the left side, followed then by the right. Intra operatively we found osteoporotic bone with cysts in both femur and tibia along with bone defects. We stabilized the tibia with long stem components. For the right side, we performed corrective osteotomy and followed it with long stem tibia components. Post op was uneventful.

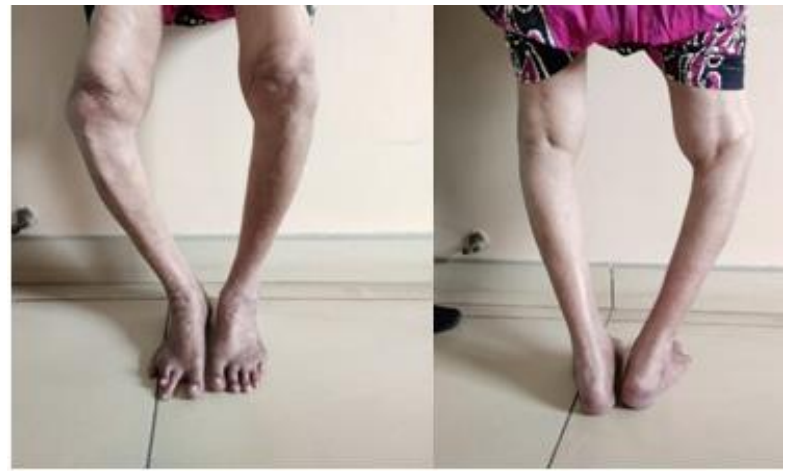

Fig 1: Pre-Op Clinical Pictures $\sim 689 \sim$ 
International Journal of Orthopaedics Sciences

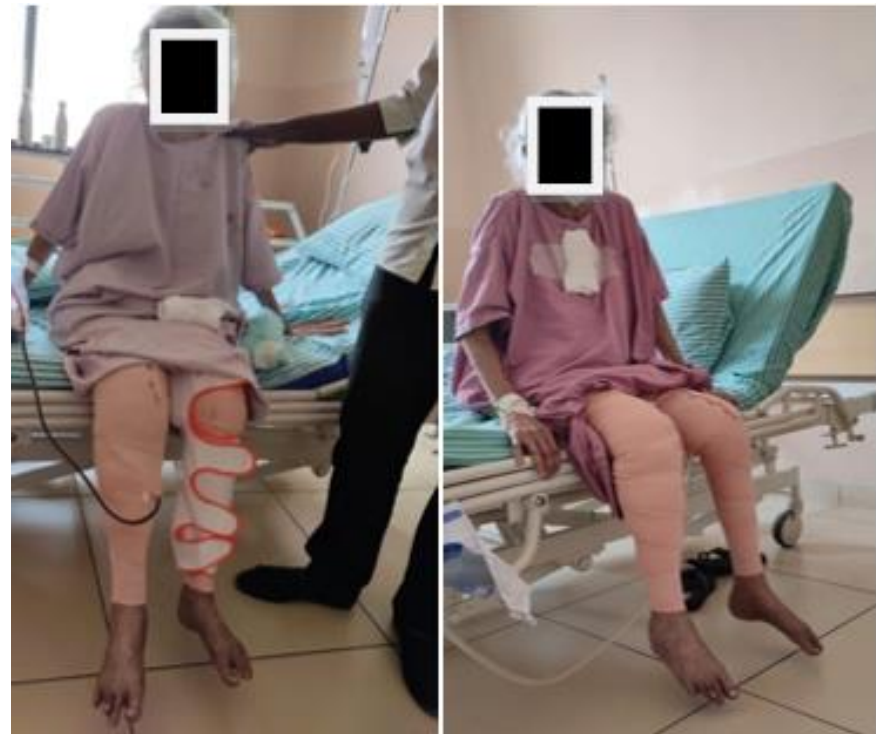

Fig 2: Post Op Clinical Pictures

\section{Results}

On table we achieved ROM of 0-110. Through our expertise and dedicated physiotherapy team we were able to achieve ROM of 90 . On Day two, patient was satisfactorily mobilized with the aid of walker.

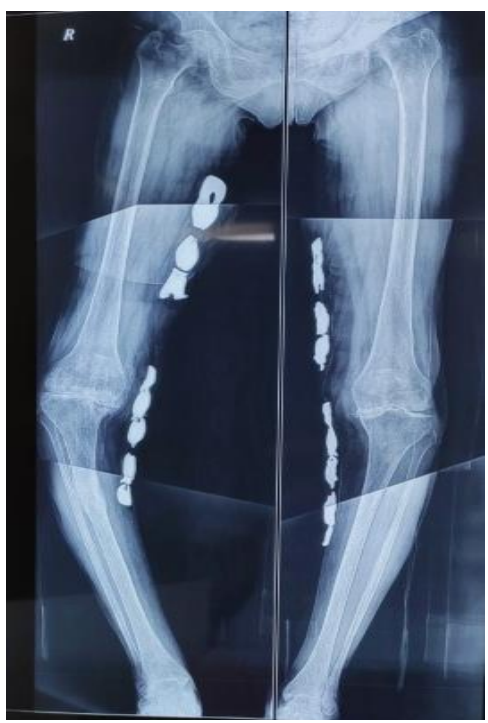

Fig 3: Scanogram

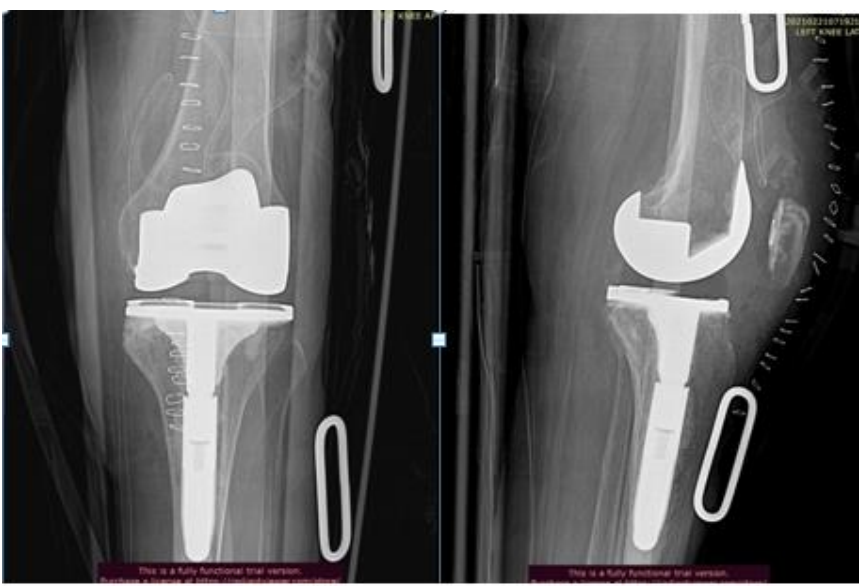

Fig 4: Rt. Post Op X-ray

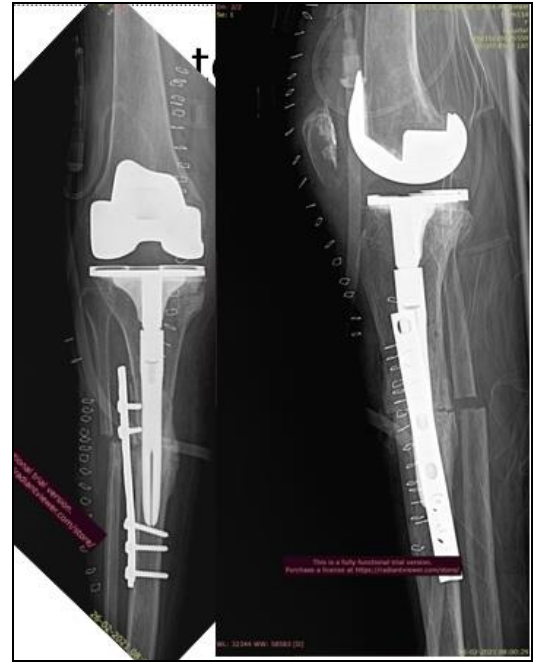

Fig 5: Lt. Post Op X-ray

\section{Discussion}

Total knee replacement gets complicated by extra-articular deformity, necessitating additional procedures in order to obtain proper anatomic and mechanical alignment of the limb. Post-traumatic deformity frequently requires careful evaluation of bone loss due malunion or non-union. Identification of the deformity at the joint line, metaphysis, or diaphysis is paramount in determining its effect on extremity alignment. This dilemma can be approached with staged procedures or via a 1-stage approach for a full correction of the deformity and alignment at the time of joint arthroplasty [2].

In this particular case, a detailed evaluation of the patient was carried out followed by pre-emptive risk assessment of challenges: Pre-op, Intra-op and Post-op respectively.

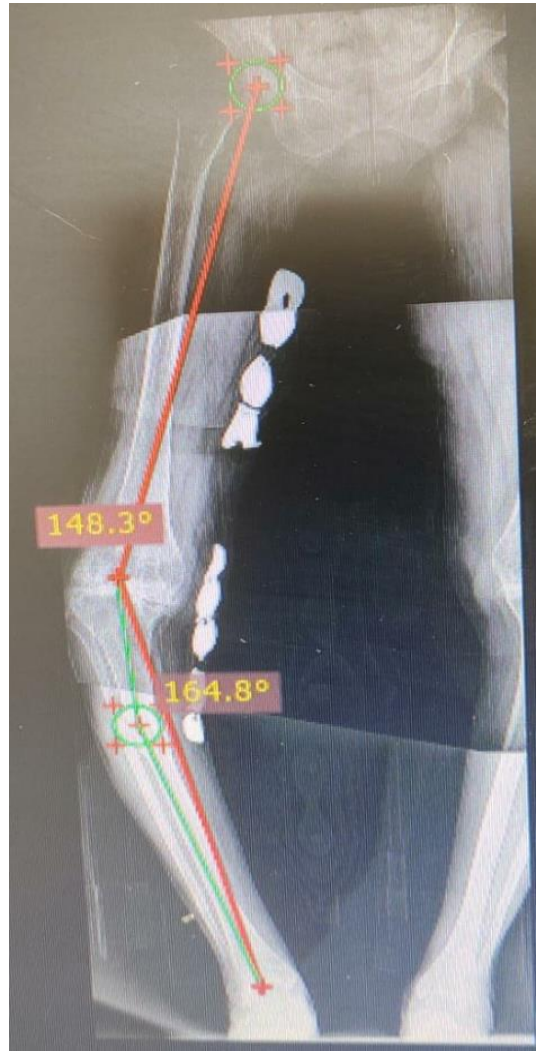

Fig 6: Calculation of Deformity wedge resection 


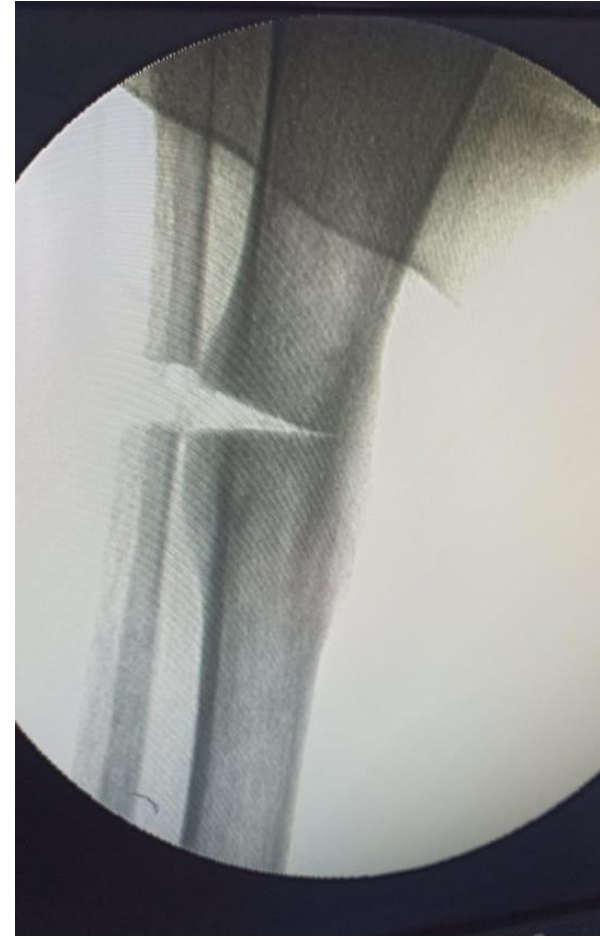

Fig 7: Intra op C-arm image showing

\section{Degree of Deformity}

Total varus deformity

Varus deformity at joint line

Proximal Malunited Tibia

After thorough evaluation and planning we proceeded for surgery. The deformity was measured at $11 \mathrm{~cm}$ from the joint line. Osteotomy site confirmed under $\mathrm{C}$-arm guidance. One centimetre of Fibula was excised and Tibia closing wedge osteotomy performed. Size of wedge was $1 \mathrm{~cm}$. Osteotomy site was stabilized by 7 holed DCP plate and bone graft. After deformity correction TKR was performed in Medial Parapatellar Approach. Both Deformity correction and TKR with application of long stem $(12 \times 150 \mathrm{~mm}$ Long stem Genesis-II, SMITH \& NEPHEW) to Tibial component was performed in a single setting so that in intra-op, a good diaphyseal axial and rotational stability could be achieved. The patient was mobilized on day two, bearing full bodily weight with the aid of a walker. Her post-op follow up was uneventful.

Radke and Radke [3] have demonstrated that total knee arthroplasty in combination with a 1-stage tibial osteotomy, has enjoyed good results. Another option to consider includes a diaphyseal osteotomy with intramedullary stem fixation, though this osteotomy type may pose a higher risk of nonunion or other complications ${ }^{[4]}$. The use of a long-stemmed tibial component was considered in this case.

A meta-analysis [5] confirmed that posterior tibial slope increased and patellar height decreased following Opening Wedge Osteotomy. Conversely, posterior tibial slope decreased and patellar height increased following Closing Wedge Osteotomy. Although both techniques led to desirable and comparable clinical results, we recommend Opening Wedge Osteotomy as an alternatively effective treatment option for selected younger patients, which then makes it easier to perform and to convert to TKA, avoids tibiofibular joint disruption and common peroneal nerve palsy, and permits multiplanar correction as compared to Closed Wedge Osteotomy. However, additional controlled studies and long- term follow-up data are required to identify ideal candidates, the type of fixation and augment selection in Opening wedge Osteotomy.

The outcome of TKR in severe varus deformity has excellent results ${ }^{[6]}$. As in our case, we have done PS knee. The outcome of the various PS knees shown survivorship of 98$100 \%$, full range of movement, great improvement in pre-op and post-op knee society scores. These results offer great encouragement to the orthopaedic fraternity world over. Combined extra-articular deformity of the tibia along with osteoarthritis of the knee-joint can present a challenging reconstructive procedure for the orthopaedic surgeon. The knee can be approached with staged procedures or via a 1stage approach for full correction of the deformity and alignment at the time of joint arthroplasty [2]. This report presents another option for a single-stage operative procedure for malunited or united proximal tibia due to stress fracture and with severe varus deformity. Additionally, concerns regarding the strength of the open wedge osteotomy site fixation device, as discussed by Stuart and colleagues ${ }^{[7]}$ may lead one to reconsider the use of our technique. Intramedullary fixation is admittedly mechanically superior and usually allows for immediate weight bearing. For this reason alone was the aforementioned patient forbidden from bearing weight until evidence of osteotomy healing was noted radiographically.

\section{Conclusion}

This case report highlights the correction of both extraarticular and intraarticular deformity correction in a single stage surgery which yields better results overall. Our experience in this case reveals single stage procedure benefits with early recovery, decreased morbidity, minimal hospital stay with best functional outcomes.

\section{References}

1. Lee JK, Choi C-H. Total Knee Arthroplasty in Rheumatoid Arthritis. Knee Surg Relat Res 2012;24(1):16.

2. Lahav A, DiMaio FR. Concurrent opening wedge osteotomy and total knee replacement in a patient with posttraumatic arthritis and a varus tibial malunion. Am J Orthop Belle Mead NJ 2007;36(8):E121-123.

3. Radke S, Radke J. Total knee arthroplasty in combination with a one-stage tibial osteotomy: a technique for correction of a gonarthrosis with a severe (>15 degrees) tibial extra-articular deformity. J Arthroplasty 2002;17(5):533-7.

4. Kaartinen E, Paavolainen $\mathrm{P}$, Holmström $\mathrm{T}$, Slätis $\mathrm{P}$, Happonen RP. Different healing patterns of experimental osteotomies treated by intramedullary nailing. Arch Orthop Trauma Surg 1993;112(4):171-4.

5. Deng Y-J, Yuan Y, Ren Y-M, Li Q, Wang D, Yang Y, et al. Differences between open-wedge versus closedwedge high tibial osteotomy on clinical and radiological outcomes and adverse events 18 .

6. Rossi R, Cottino U, Bruzzone M, Dettoni F, Bonasia DE, Rosso F. Total knee arthroplasty in the varus knee: tips and tricks. Int Orthop 2019;43(1):151-8.

7. Stuart MJ, Beachy AM, Grabowski JJ, An KN, Kaufman KR. Biomechanical evaluation of a proximal tibial opening-wedge osteotomy plate. Am J Knee Surg 1999;12(3):148-53. discussion 153-154. 